\title{
Écroulements et mouvements de versants associés au niveau du col de Onsar (Rif, Maroc) dans un contexte géologique de décrochement
}

Y. EL KHARIM

C. DARRAZ

R. HLILA

K. EL. HAJJAJI

Faculté des sciences

Université Abdelmalek

Essaadi,

Mhannech II. BP 2121

Tétouan (Maroc)

e-mail : ykharim@fst.ac.ma
Le col de Onsar est un accident morphologique qui sépare les deux tronçons de la chaîne calcaire du Haouz. La nature et l'origine de ce col sont à rattacher à son contexte tectonique et à son évolution géomorphologique récente. Il représente, du fait de l'intense déformation tectonique, un couloir de fragilisation des matériaux qui se traduit par des phénomènes d'instabilité aussi nombreux que variés. Dans ce contexte, un village qui porte le nom du col a connu en 1946 et 1963 des écroulements rocheux entraînant des pertes humaines et matérielles. Le village est bâti sur une ancienne accumulation de blocs et la présence d'indices d'instabilité actuels atteste du risque permanent d'écroulement. Ces événements du village ne sont pas des cas isolés puisqu'un inventaire des mouvements de versants dans les environs du col de Onsar révèle la présence d'une instabilité assez importante, dont l'origine et le mécanisme de mise en place sont à rechercher dans le contexte géologique qui est particulièrement complexe.

Mots-clés : mouvements de versant, écroulements, décrochement, col de Onsar, Rif.
NDLE: Les discussions sur cet article sont acceptées jusqu'au $1^{\text {er }}$ novembre 2003
The Onsar Pass is a morphological accident separating the two parts of the El Haouz limestone chain. The nature and origin of this pass are related to the tectonic setting and the recent geomorphological evolution. Through an intense deformation, it constitutes a corridor of weakened materials which results in phenomena of instability that range from sagging and rockfalls in the karstic carbonate formations to creeping phenomena and flows in the underlying schistouse and marly formations. in this regard, a village bearing the same name as the pass witnessed rockfalls in the winter of 1946 and 1963 which caused the loss of life and goods. The basement of the village is an old accumulation of blocks, and the presence of signs of instability attests to the persistent risk in this site. This event as well as its aftermath are not an isolated case. In fact, an inventory made in the surrounding of the Onsar Pass has shown the presence of numerous and varied slope movements. Therefore, this process generalize this unstable environment to the entire area of the pass, the origin and mechanism of which can be explained by the geological context, which is particularly complex.

Key words : slope movements, rockfalls, strike-slip fault, Onsar pass, Rif. 


\section{Introduction}

Le col de Onsar, situé à $7 \mathrm{~km}$ au nord de Tétouan, est un accident morphologique qui sépare deux tronçons de la chaîne calcaire du Haouz (Fig. 1). Il abrite un village du même nom qui a connu en 1946 et 1963 des écroulements entraînant des dégâts humains et matériels (El Kharim et al., 2001). Ce village est installé, comme bien d'autres, au pied de la chaîne carbonatée, au contact entre des formations karstiques et des terrains imperméables sous-jacents. Ce type d'habitat s'explique par la proximité des émergences de sources d'origine karstique, d'oú le nom de Onsar quỉ désigne en dialecte local une source à débit important, surtout en hiver.

Le caractère évolutif et rẻcurrent de l'écroulement du village de Onsar et la fréquence élevée des mouvements de versants observés à l'échelle du col, justifient amplement l'évaluation des conditions d'instabilité. Ces dernières sont étroitement liées à la structure géologique et à l'évolution géomorphologique du col de Onsar,

\section{Géologie du secteur}

La zone étudiée est située dans le Rif septentrional, oủ la chaîne rifaine acquiert une disposition N-S, juste au niveau des contacts géologiques majeurs entre les grands domaines de la chaine (Fig. 2) : les nappes Ghomarides à matériel paléozoïque à l'est, la chaine calcaire ou « dorsale calcaire » (Fallot, 1937) à matériel essentiellement secondaire au centre et le domaine des nappes de flyschs tertiaires à l'ouest. La succession de déformations compressives et distensives alpines, appliquée à un matériel hétérogène (alternance de niveaux rigides et déformables), a généré une structure particulièrement complexe dont dépendent largement les mouvements de versants, sujets de cette étude.

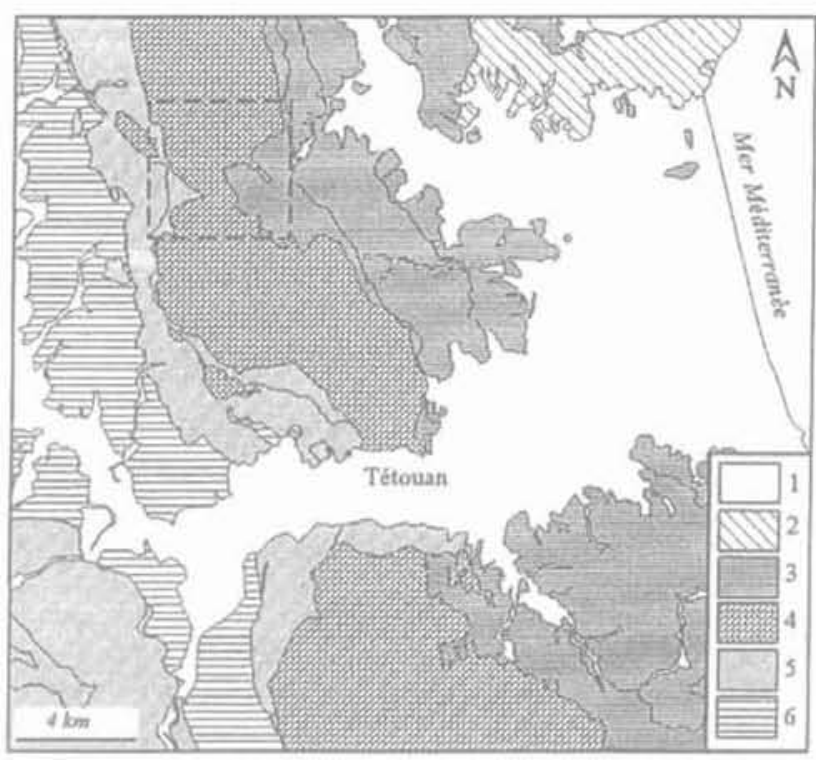

Fis. 2 .

Cadre géologique. Post-nappe (1) ; zones internes : Sebtides (2) ; Ghomarides (3) ; dorsale calcaire (4); nappes des flyschs (5) ; zones externes : unité de Tanger (6). Geological setting. Postorogenics (1) ; internal zones : Sebtides (2), Ghomarides (3), limestone chain $"$ dorsale calcaire $\#(4)$; flysch nappes (5) : external zones: Tangier unit (6).

\section{Aperçu lithostratigraphique}

La dorsale calcaire est formée de plusieurs unités ou écailles structurales (Fig. 3) qui montrent toutes une formation carbonatée massive de base. Dans le secteur étudié, cette dernière consiste en des dolomies grises triasiques, dont la puissance atteint par endroit $150 \mathrm{~m}$, et en des calcaires massifs blancs de l'Hettangien aussi puissants que les premières. Dans le cas de l'unité de Belouazen (BO) et de Uestia 1 (U1), ces formations car-

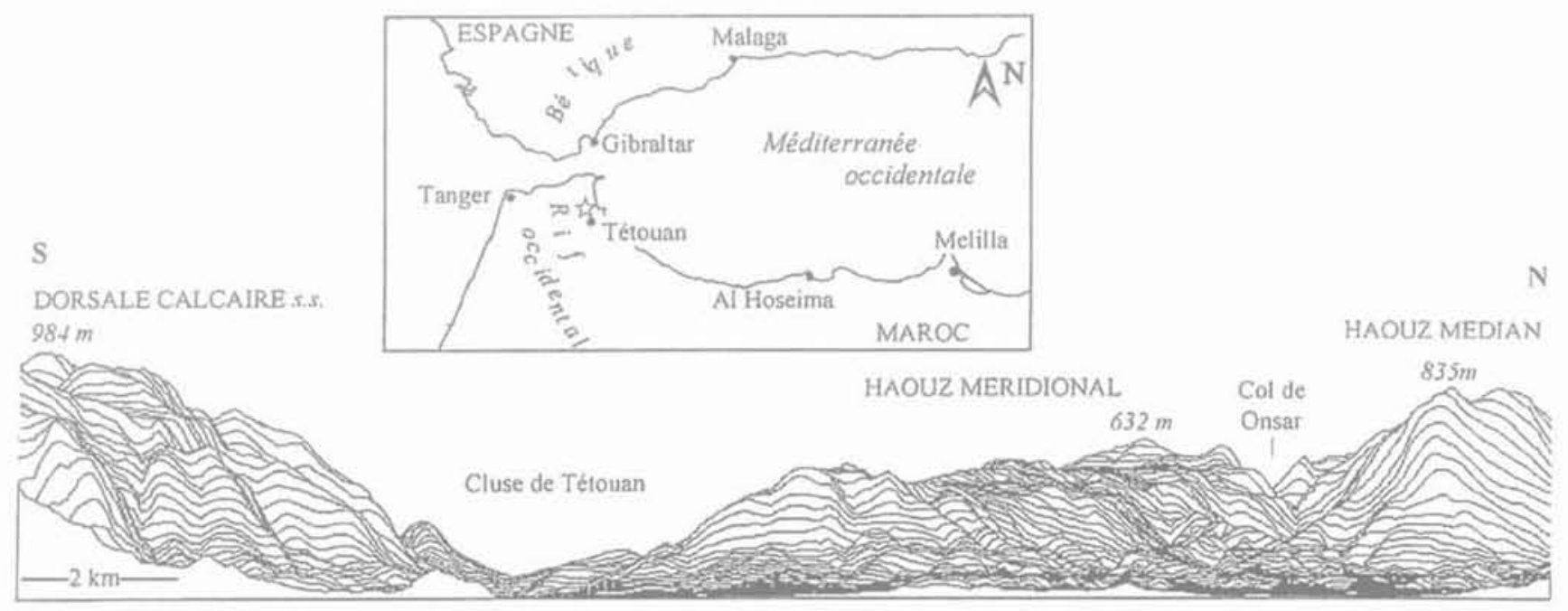

FIG.1 Localisation géographique du secteur d'étude et profil topographique N-S. Location of the study area and the N-S topographic profil. 
bonatées massives succèdent, en continuité stratigraphique, aux grès et argilites rouges du Permo-Trias. Cependant, la différence entre les différentes écailles de la dorsale calcaire réside surtout dans leurs termes Jurassico-Crétacé, constitués de quelques mètres de séries condensées (calcaires lités à silex, radiolarites, calcaires marneux...). La couverture tertiaire de la Dorsale, peu épaisse, est représentée par des marnes éo-oligocènes jaunes à passées bioclastiques à nummulites. Ces marnes affleurent par endroit en liserés entre les écailles ou sous les massifs carbonatés sus-mentionnés.

La nappe Ghomaride de Koudiat Tizian (Milliard in Durand-Delga et al., 1962) affleure, à l'est de la Dorsale calcaire, sous forme de schistes et grauwackes, plissotés et schistosés, d'âge paléozoíque et de grès et argilites rouges permo-triasiques. A l'ouest, les nappes de flyschs tertiaires qui supportent en contact sub-vertical la Dorsale calcaire, sont formées d'alternances marnocalcaires (nappe de Béni Ider, Durand-Delga et al. 1962) et marno-gréseuses (le Prédorsalien, DurandDelga, 1972).

Le Quaternaire y est représenté essentiellement par des dépôts détritiques, produits du démantèlement des reliefs et de la dynamique des versants (éboulis, dépôts de mouvements de masse, colluvions, cônes de déjection) et sporadiquement par des travertins qui le plus souvent s'observent au contact dorsale externe/Flyschs à Keddana.

\section{2}

\section{Cadre structural}

L'histoire structurale polyphasée du secteur s'échelonne principalement du Secondaire au Tertiaire. Cependant, ce sont les événements compressifs paroxysmaux de l'Aquitanien-Burdigalien qui ont marqué l'essentiel de l'histoire géologique de la région. Ces événements correspondent à des chevauchements à vergence ouest et à des rétrochevauchements associés vers l'est. Ils sont responsables des grandes structures sub-méridiennes, plissées, écaillées et disposées en éventail, de la chaine calcaire du Haouz (Raoult, 1966). Un ensemble de failles et accidents à caractère décrochant dont celui de Onsar vient achever la structuration par l'individualisation des tronçons de la chaîne calcaire : direction sub-méridienne du Haouz médian et NO-SE du Haouz méridional.
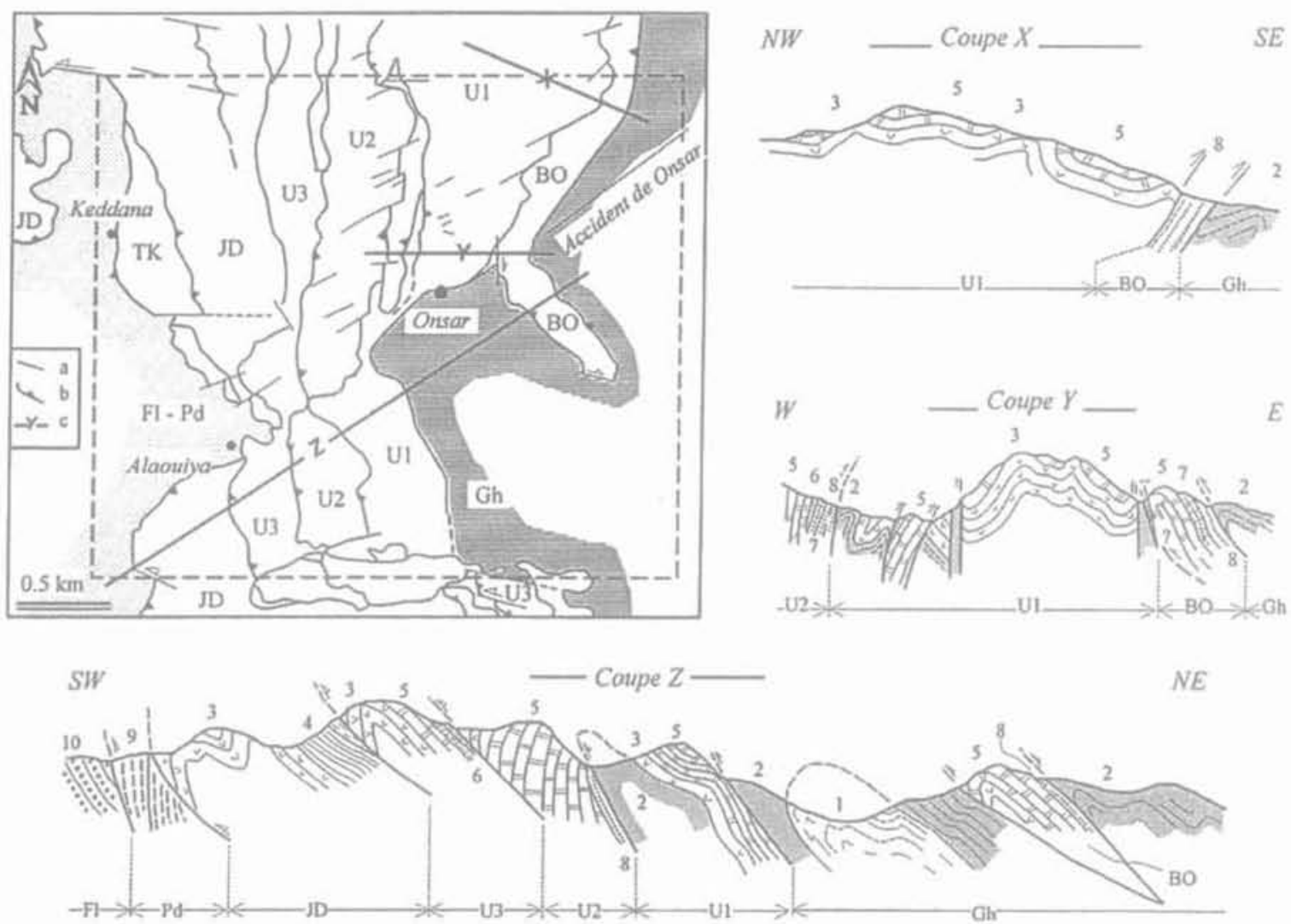

Schéma structural.

Faille décrochante (a), chevauchement (b), coupe géologique (c) ; Ghomarides (Gh) ; unités tectoniques de la dorsale calcaire : Belouazen (BO), Uestia 1 (U1), Uestia 2 (U2), Uestia 3 (U3), Jbel Dersa (JD), Tarrant-El Keddan (TK) ; Prédorsalien (Pd) ; Béni Ider (FI) ; schistes et grauwackes (1), grès et argilites rouges (2), dolomies (3), séries calcaro-dolomitiques (4), calcaires massifs (5), calcaires à silex (6), calcaires organogènes (7), marnes et conglomérats éo-oligocènes (8), flyschs à alternance de grès quartzeux et marnes (9), alternance de marnes et de bancs calcaires (10).

Structural scheme.

Strike-slip fault (a), thrust fault (b), geological section (c) : Ghomarides (Gh) ; tectonic units of the Limestone chain : Belouazen (BO), Uestia 1 (U1), Uestia 2 (U2), Uestia 3 (U3), Jbel Dersa (JD), Tarrant-EI Keddan (TK) ; Nappes of flyschs : Predorsalian (Pd) : Beni Ider (Fl) : schists and greywacks (1), red sandstones and clays (2), massive dolomites (3), limestones and dolomites series (4), massive limestones (5), limestones with silex (6), bioclastic marly limestones (7), yellow marls and conglomerates (8), marls with sandstone (9), red marls with limestone beds (10). 
D'échelle plurikilométrique et bien visible sur les ortho-images au 1/100 000 du détroit de Gibraltar (MOPT, 1991), l'accident de Onsar affecte aussi bien le socle que sa couverture. Il prend naissance dans les nappes paléozoïques à l'est, traverse tout le chaînon du Haouz et continue à l'ouest dans le domaine des flyschs (El Mrihi, 1995). Il s'agit d'un décrochement dextre $\left(\mathrm{N} 50^{\circ}-60^{\circ}\right.$ ), comme en témoigne la torsion anti-horaire des structures d'échelle cartographique au sud immédiat de cet accident dont le jeu peut atteindre un kilomètre (Hlila, 1994). Des décrochements sénestres, $\mathrm{N} 110^{\circ}-130^{\circ}$, constituent, avec la famille $\mathrm{N} 50^{\circ}-60^{\circ}$, un système de failles conjuguées. L'analyse de la microfracturation révèle également l'existence de quatre familles de diaclases (Tableau I).

\begin{tabular}{|c|c|c|c|}
\hline TABLEAUI & \multicolumn{3}{|c|}{$\begin{array}{l}\text { Caractéristiques des principales familles } \\
\text { de fractures. } \\
\text { Characteristics of the main fracture families. }\end{array}$} \\
\hline & Direction & Pendage & Type \\
\hline Famille a & $N 50^{\circ}-60^{\circ}$ & $90^{\circ}-75^{\circ} \mathrm{SE}$ & faille, diaclase \\
\hline Famille b & $N 75^{\circ}-90^{\circ}$ & $90^{\circ}-80^{\circ} \mathrm{S}$ & diaclase \\
\hline Famille c & $N 105^{\circ}-130^{\circ}$ & $90^{\circ}-80^{\circ} \mathrm{NNE}$ & faille, diaclase \\
\hline Famille d & $N 340^{\circ}-20^{\circ}$ & $90^{\circ}-80^{\circ} \mathrm{E}$ & diaclase \\
\hline
\end{tabular}

A l'emplacement du village de Onsar, les marnes éo-oligocènes de l'unité de Belouazen ( $\mathrm{BO}$ ) se trouvent biseautées et entrainées à l'ouest par ce mouvement décrochant, sous les dolomies et calcaires massifs de I'unité Uestia 1 (U1). Cette disposition structurale des marnes tendres sous les formations massives de l'unité tectonique voisine est à l'origine de l'écroulement du village de Onsar. A l'ouest de ce village, les unités internes de la dorsale calcaire se pincent fortement et se réduisent à quelques lambeaux disloqués et orientés parallèlement à la direction de l'accident dont le pendage local est subvertical.

Un ensemble d'événements tectoniques postparoxysmaux de moindre importance, dont les effets se poursuivent jusqu'à la période actuelle, se matérialise principalement par le rejeu normal des accidents antérieurs. En l'absence d'études précises, les séismes de forte magnitude qui ont affecté la région durant les siècles passés, probablement à l'origine d'anciens glissements de grande ampleur et la sismicité modérée actuelle sont les seuls indices de l'existence d'une tectonique active.

\section{3}

\section{Géomorphologie}

Conséquence d'une telle structuration, le relief y est très escarpé, allant de $120 \mathrm{~m}$ au niveau de l'oued El Lil à $835 \mathrm{~m}$ sur les hautes crêtes calcaro-dolomitiques (Haouz médian), soit une dénivelée totale supérieure à $700 \mathrm{~m}$. Au sud, les hauteurs atteignent à peine $600 \mathrm{~m}$ d'altitude (Fig. 1). C'est principalement le raccourcissement de la chaîne du Haouz, estimé à $75 \%$ d'après les reconstructions palinspatiques (Maate, 1996), qui aurait généré les grands reliefs situés au nord du village de Onsar dans lesquels les failles décrochantes dextres délimitent des tronçons du massif carbonaté descendant en gradins vers le col. Les pentes y sont relativement très importantes : $18^{\circ}$ en moyenne dans les grès et argilites rouges du Permo-Trias et les schistes des Ghomarides, $34^{\circ}$ entre les écailles de la dorsale et plus de $63^{\circ}$ dans le cas des falaises rocheuses escarpées.

Les ravins creusés dans les grès et argilites permotriasiques et surtout dans les schistes sous-jacents mettent à jour plusieurs mètres de matériau s'altérant facilement en surface. Ce processus est accéléré par l'activité agricole, produisant un sol très argileux, instable lorsqu'il est gorgé d'eau. Les terrains carbonatés caractérisés par un relief ruiniforme, surtout dans les dolomies plus altérables, sont le siège de phénomènes karstiques, comme l'atteste la présence de dolines et grottes dans le secteur. Les dolines ont été observées sous différentes tailles, alignées suivant les contacts des unités structurales alors que les grottes sont localisées au niveau des crètes carbonatées à la faveur de fractures transverses. A la base de ces formations carbonatées karstiques, reposant sur des formations imperméables, se rencontrent les résurgences et les sources.

\section{4}

\section{Phénomènes d'instabilité au niveau du col de Onsar}

Une analyse des photographies aériennes au 1/20000 des missions 1966 et 1986 avec vérification sur le terrain ont permis de recenser 65 mouvements sur une surface totale approximative de $9 \mathrm{~km}^{2}$, soit 7 par $\mathrm{km}^{2}$ (Fig. 4). Ces mouvements de versants présentent un certain nombre de caractères spécifiques, notamment : (i) leur nombre élevé sur une superficie relativement réduite; (ii) la continuité des mouvements dans le temps car certains se sont manifestés à plusieurs reprises et peuvent encore évoluer de nos jours ; (iii) l'existence simultanée en un même lieu de processus de rupture de types plastique et cassante et (iv) leur alignement sur les accidents géologiques, notamment celui de Onsar (NE-SO).

Les glissements, coulées et solifluxions, relativement peu abondants et de petites tailles, affectent les marnes, les flyschs et les colluvions qui les surmontent. Cependant, l'aspect tout à fait caractéristique est la concomitance de ces processus de type plastique avec les écroulements dans les matériaux carbonatés susjacents. Ces associations sont observables lorsque le pied des falaises est dégagé d'éboulis (Fig. 5).

Les écroulements par simples chutes de blocs ou éboulements en masse sont les plus abondants dans le secteur. Ils dégagent des falaises hautes de plusieurs dizaines de mètres. Les surfaces de rupture sont prédéfinies par la direction, le pendage et la densité des cassures (diaclases et failles) du massif carbonaté (Fig. 6). Certaines falaises montrent également des masses rocheuses effondrées par tassements suivant des diaclases verticales, parallèles et transverses à l'escarpement principal. 


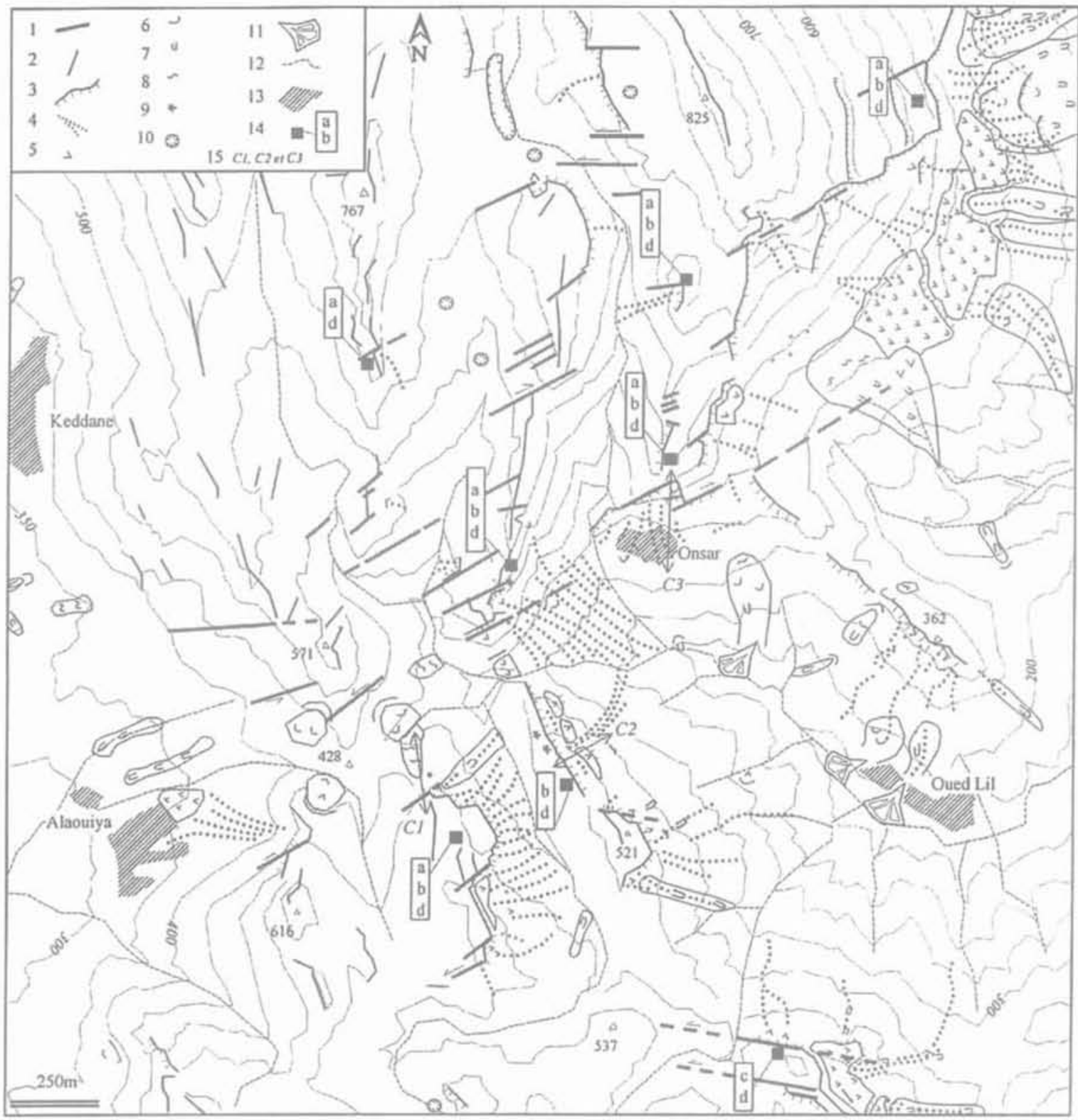

FIG.4 Mouvements de versants dans le col de Onsar.

Failles (1), crête (2), falaise escarpée (3) ; écroulement (4) ; tassement rocheux (5) ; glissement (6) ; coulée (7); solifluxion (8); grotte $(9)$; doline $(10)$; cône de déjection (11); réseau hydrographique (12); village (13); famille de fractures (14), localisation des coupes schématiques des figures 5, 6 et 8 (15).

Slope movements in the Onsar pass.

Fault (1), crest (2), steep scarp (3), rockfall (4), sagging rocky panels (5), slide (6), flow (7), solifluxion (8), karstic cave (9), dolines (10), detritical fan (11), hydrography (12), village (13), joint families (14), location of schematic cross-section of figures 5,6 and $8(15)$.

C'est dans sa partie nord-est que le secteur montre une instabilité complexe et de grande ampleur, en raison de la double influence structurale qu'a subie cette zone : un compartimentage décrochant compliqué par le rétrochevauchement en relief accusé de la partie interne de la dorsale sur les Ghomarides, dont la limite est soulignée par les marnes éo-oligocènes jaunes de l'unité de Belouazen (BO). Les résurgences, au niveau de ce contact, abaissent la résistance des marnes, et leur infiltration dans les masses carbonatées affaissées déstabilise de nouveau ces dernières, en les impliquant dans des mouvements de type glissement rocheux-coulée de blocs et débris.

Les schistes et grauwackes paléozoïques se montrent relativement stables. Toutefois, les photographies aériennes de 1966 ont permis d'identifier d'anciens glis- sements dont les masses, très peu déplacées, se vident progressivement et alimentent les petits cônes de bas de pente. Par ailleurs, les témoignages des habitants font état de petites coulées de débris sporadiques, par décollement de la partie superficielle altérée, formant de petits reliefs que les activités de labour estompent avec le temps. Des phénomènes de reptation se produisent également dans ces matériaux paléozoïques, comme en témoignent les troncs déformés des figuiers.

\section{5}

\section{Écroulements du village de Onsar}

En novembre 1945, des pluies exceptionnellement importantes ont provoqué un mouvement complexe de 


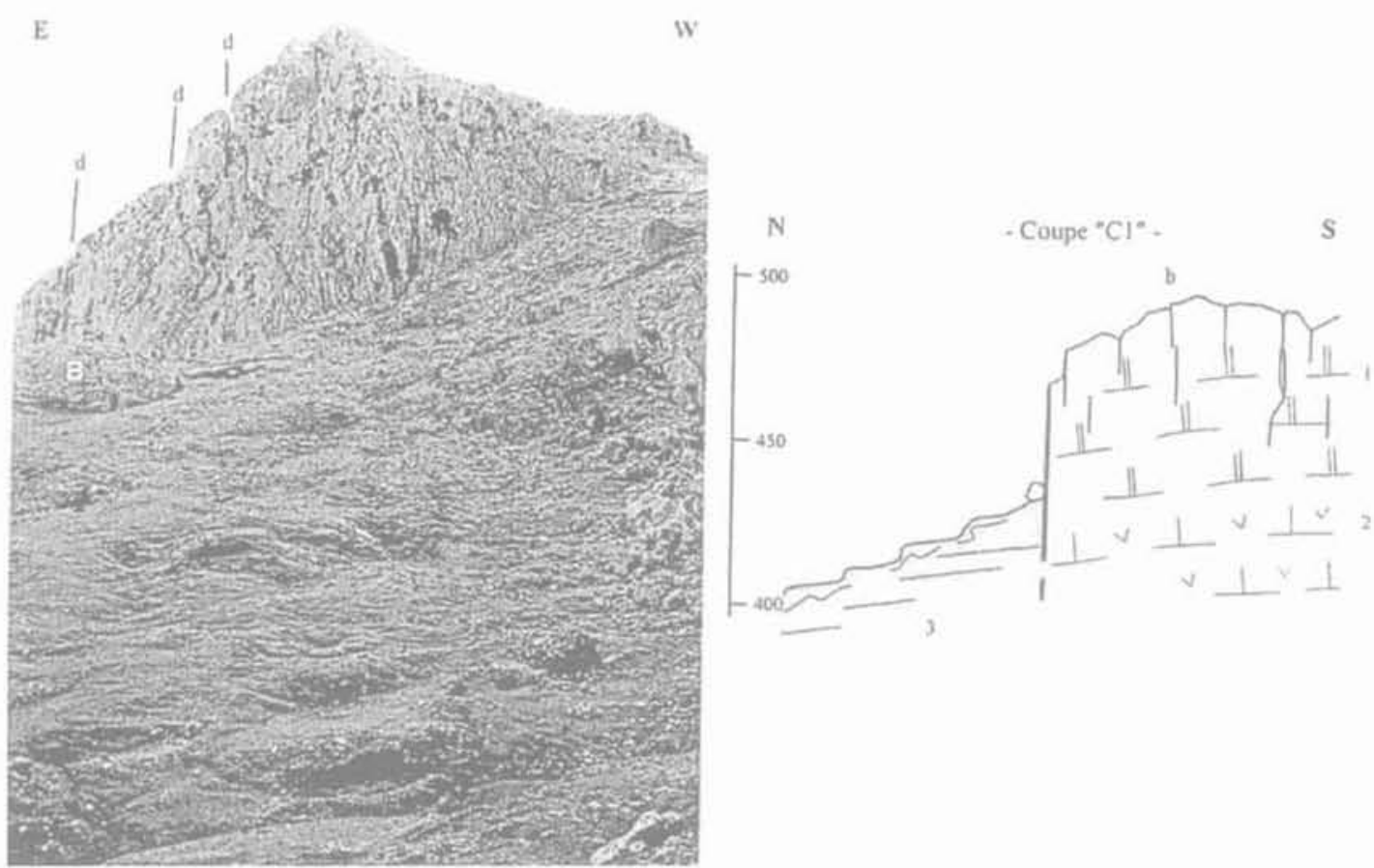

FiG.5 Solifluxion dans la formation rouge permo-triasique sous un escarpement rocheux très diaclasé.

Bloc écroulé (B) ; familles « b » et « d » de diaclases (b) et (d) ; unité structurale U2 : calcaires massifs (1), dolomies massives (2); unité structurale U1 : argilites et grès rouges (3).

Solifluxion in the red clays and sandistones formation under the active scarp of limestone.

Falled limestone block (B); $\mu \mathrm{b}, \mathrm{a}$ and $\| \mathrm{d} x$ joint families (b) and $(\mathrm{d})$; U2 structural unit : massive limestones (1), massive dolomites (2); U1 structural unit : red sandstones and clays (3).

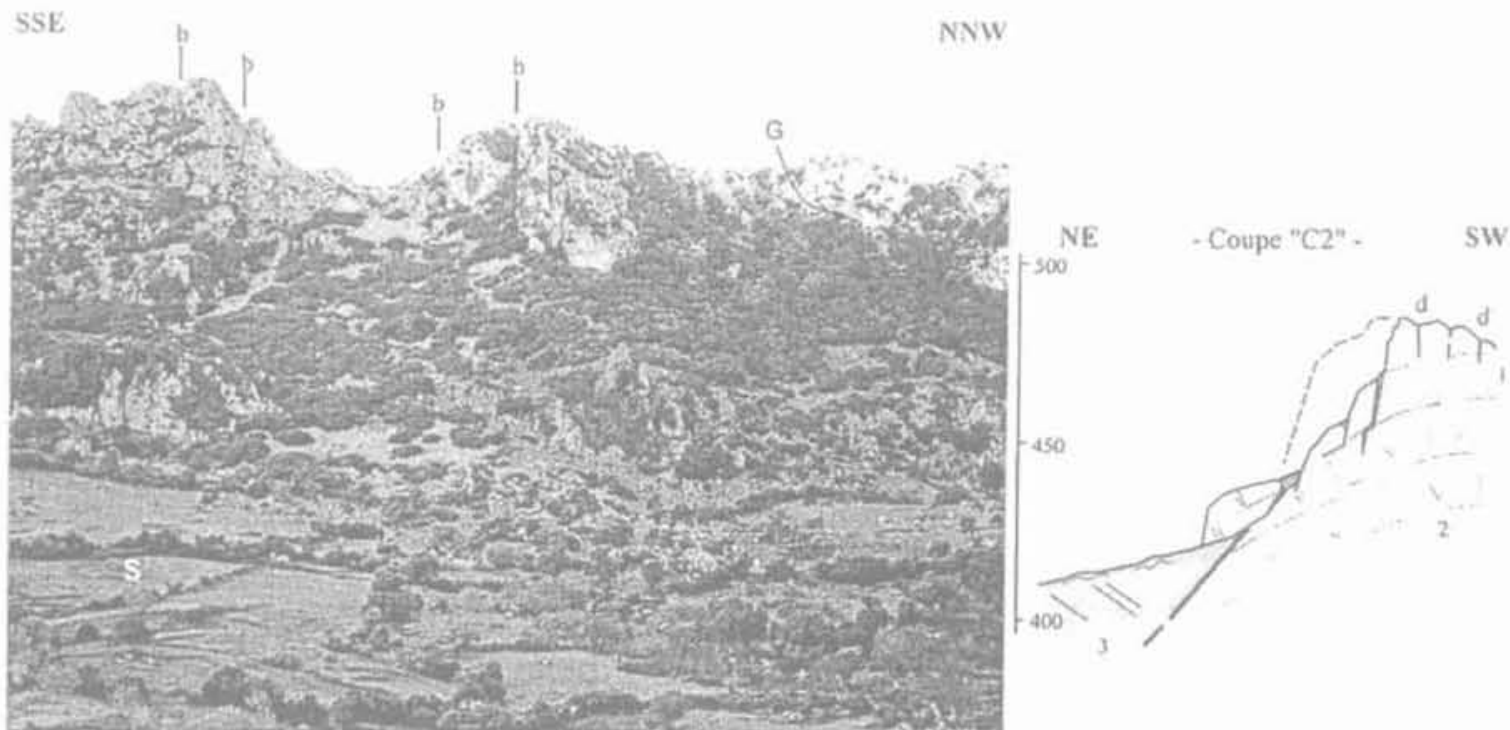

Fị. 6 Contrôle structural d'un écroulement ancien.

Familles $\alpha$ b $)$ et $\propto$ d $)$ de diaclases (b) et (d) ; grotte karstique (G) ; solifluxion (S) ; unité U1 : calcaires massifs (1), dolomies massives (2); Ghomarides : grès et argilites rouges (3).

Structural control of an old rockfall.

$a b s$ and $a d n$ joint families (b) et (d) : karstic cave (G) ; solifluxion (S) ; U1 unit : massive limestones (1), massive dolomites (2) ; Ghomarides : red sandstones and clays (3).

type glissement-coulée dans les assises marneuses éooligocènes de l'unité BO, ce qui a placé l'escarpement carbonaté de l'unité U1 en surplomb. Les habitants du village ont pu observer le basculement progressif et lent du pan rocheux en surplomb, mais l'écroulement final les a surpris de nuit trois mois après (février 1946), ensevelissant une partie du village, entraînant la mort de cinq habitants et des pertes matérielles importantes. Des chutes de blocs durant l'hiver pluvieux de $1962-$ 1963 ont de nouveau causé des dégâts. Dans les deux cas, les conditions climatiques responsables sont des précipitations importantes comme en attestent les rele- 
vés de la station météorologique de la ville de Tétouan (Fig. 7). Celle-ci, située à faible altitude, avait enregistré, durant la saison hivernale (de novembre à février) de ces deux années hydrologiques, des valeurs qui dépassent la moyenne annuelle calculée sur 82 ans. Compte tenu de la moyenne mobile sur deux ans, ces deux événements coïncident avec des périodes bi- à triannuelles exceptionnellement pluvieuses. Notons, enfin, que la relative sécheresse du climat que connaît récemment la région, illustrée par la moyenne mobile calculée sur dix ans, pourrait bien expliquer l'absence de réactivations constatées durant les trois dernières décennies,

Ces deux événements n'ont en fait concerné qu'une partie de la zone de rupture totale qui dépasse $50 \mathrm{~m}$ de hauteur, $250 \mathrm{~m}$ de largeur et $80^{\circ} \mathrm{d}$ 'inclinaison moyenne, zone dont la morphologie est délimitée sensiblement par les plans N50, NS et EO. L'examen détaillé, en arrière et au niveau de cette zone de rupture, montre un découpage du massif rocheux par trois familles principales de discontinuités ( $\mathrm{a}, \mathrm{b}$ et d) auxquelles s'ajoute le contact stratigraphique subhorizontal (légèrement incliné dans le sens de la pente) entre les dolomies grises et les calcaires massifs supérieurs. La zone de rupture montre, par ailleurs, un pan rocheux tassé et suspendu sous la niche d'arrachement principale. Les assises marneuses jaunes de base, responsables du fluage basal, sont masquées par un cône d'éboulis dont l'angle d'inclinaison est de $26,5^{\circ}$ et sur lequel sont parsemés des blocs de taille métrique. Des échantillons des marnes éo-oligocènes prélevés à l'est du village oủ elles affleurent, montre les caractéristiques suivantes: les deux échantillons de surface (à $0,30 \mathrm{~m}$ de profondeur) ont montré un comportement plastique dominant (teneur en eau naturelle $\mathrm{W}=24 \%$ et $21,5 \%$, limite de liquidité WL $=50,5 \%$ et $53 \%$ et indice de plasticité $\mathrm{IP}=27,4 \%$ et $30,5 \%$ ) et une cohésion apparente, par Yessai de cisaillement direct, de 0,008 MPa et 0,017 MPa. En profondeur, à $1,85 \mathrm{~m}$, les caractéristiques géotechniques s'améliorent notablement $(\mathrm{W}=9 \%$ et $5 \%$, $\mathrm{WL}=22 \%$ et $35 \%, \mathrm{IP}=16$ et $23 \%, \mathrm{C}_{\mathrm{u}}=0,115 \mathrm{MPa}$ et $0,175 \mathrm{MPa})$.

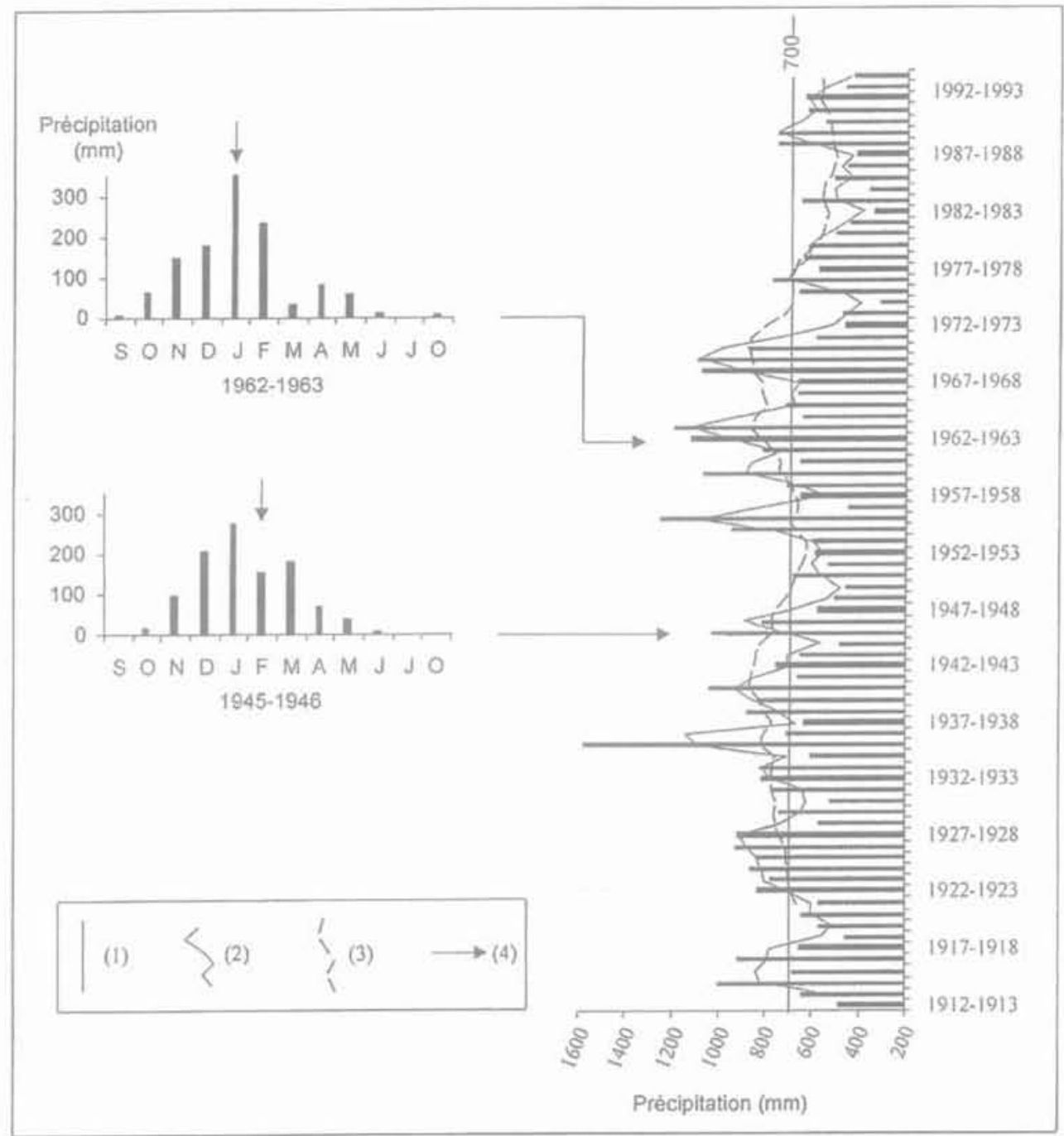

Évolution des précipitations annuelles et mensuelles entre 1912 et 1995 (station météorologique de Tétouan) et la localisation temporelle des réactivations de l'écroulement de Onsar.

Moyenne annuelle (1), moyenne annuelle mobile sur 2 ans (2), moyenne annuelle mobile sur 10 ans (3), localisation temporelle des réactivations de l'écroulement du village de Onsar.

Annual and monthly rainfall evolution between 1912 and 1995 (case of the meteorological station of Tetuan) and references of Rockfall events of the Onsar village

Annual average (1), sliding average on 2 years (2), sliding average on 10 years (3), time localisation of the Onsar rockfall events. 
Le village est bâti sur une accumulation de débris de blocs dépassant la taille des maisons. La majeure partie de l'écroulement s'est réalisée bien longtemps avant l'installation du village. L'extension limitée des dépôts dans le sens de la pente suggère que cette ancienne masse écroulée correspond à un glissement rocheux avec désagrégation modérée. Par la suite, cette masse a pu jouer le rôle d'obstacle pour les blocs détachés postérieurement, comme ce fut le cas pour les deux événements catastrophiques de 1946 et 1963.

\section{Causes et évolution de l'instabilité}

Il convient de souligner que l'essentiel des mouvements ci-dessus n'est pas récent comme en attestent les photos aériennes et les témoignages locaux. Les masses glissées et affaissées, très dégradées et de grande ampleur, observées au NE du secteur, sur le contact dorsale calcaire/Ghomarides semblent être très anciennes. Néanmoins, la présence de diaclases béantes ou de pans rocheux isolés sur presque tous les escarpements rend compte d'un état d'instabilité actuel, de phénomènes d'évolution régressive et de la pérennité de l'aléa dans le secteur.

La décharge diffuse et continue des aquifères à travers les contacts entre matériaux compétents (calcaires, dolomies soumis à un régime karstique) et incompé- tents (marnes, argilites et schistes facilement altérables) crée les conditions de déclenchement de phénomènes de solifluxions, de glissements ou de coulées, dont les intensités sont fonctions des degrés de saturation et d'altération des matériaux. Les phénomènes de fluage dans les matériaux imperméables créent des conditions de surplomb des matériaux rocheux sus-jacents. Selon l'orientation des discontinuités et du réseau karstique et sous l'effet des pressions interstitielles dans les fissures, ces masses rocheuses subissent des tassements ou des écroulements. La propagation, la mise en place et la forme des masses écroulées dépendent de la morphologie du substratum : un replat en bas de falaise freine la masse écroulée, tandis que les fortes dénivelées donnent des amas étalés le long des pentes ou conduisent à un couloir de débris.

Compte tenu de l'activité sismique régionale modérée des dernières décennies, le rôle de l'eau semble ètre déterminant au moins dans le cas des mouvements récents. L'eau agit comme facteur déclenchant, suite à des précipitations exceptionnelles. Cependant, on ne peut écarter le rôle de la sismicité dans le déclenchement des grands mouvements et écroulements anciens de ce secteur, puisque la région a connu au cours des siècles passés des séismes de forte magnitude, le dernier en date remonte à 1909 (intensité VIII dans l'échelle MSK). Signalons que la chaine calcaire de Bokoya, à l'ouest d'El Hoceima, équivalent oriental de la Dorsale calcaire, a connu de nombreux effondrements de falaises lors du séisme de 1848 (Margaa, 1994).

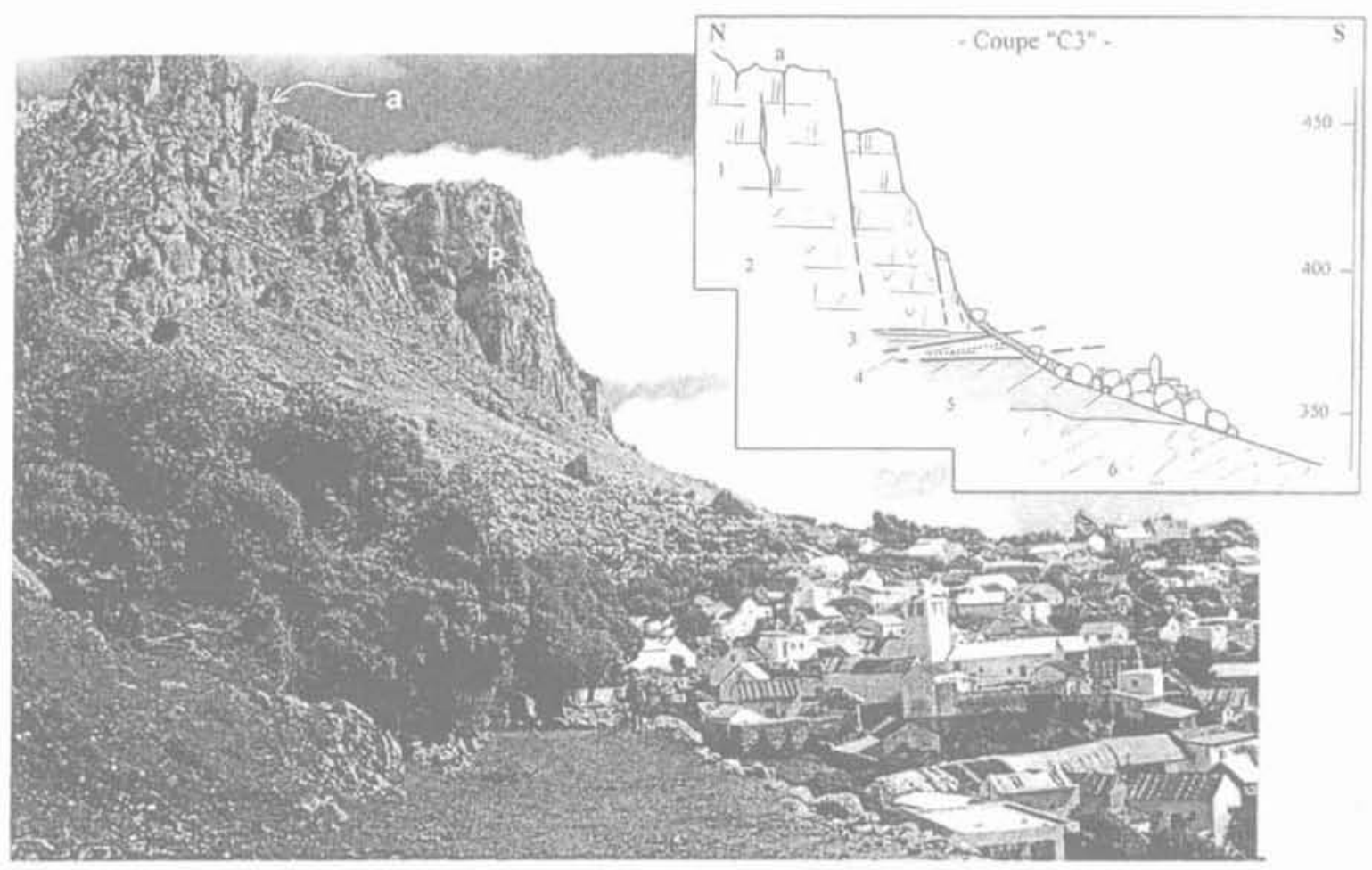

FIG 8 Écroulement du village de Onsar.

Pans rocheux tassés (P), famille ( $a$ ) de diaclases (a) ; unité U1 : calcaires massifs (1), dolomies massives (2), grès et argilites rouges (3) ; unité BO : marnes tertiaires (4) ; Ghomarides : grès et argilites rouges (5), schistes et grauwackes (6). Rockfall of the Onsar village.

sagging rocky panel (P), «a » joint family (a) : U1 unit : massive limestones (1), massive dolomites (2), red sandstones and clays (3) : BO unit : tertiary marls (4) : Ghomarides : red sandstones and clays (5), schists and greywacks (6). 


\section{Conclusion}

Le col de Onsar est affecté de nombreux mouvements de versants favorisés, en périodes de pluies abondantes, par les conditions lithologiques et structurales régionales. Il faut remarquer une diversité typologique résultant de mécanismes très différents, puisque l'on observe séparément ou en concomitance : des coulées, des solifluxions, des glissements, des tassements auxquels se surimposent des écroulements et des chutes de blocs. Le caractère catastrophique des événements du village de Onsar, survenus en 1946 et 1963 , n'est dû qu'á la coïncidence de l'aléa et des enjeux, car, de par le volume des blocs et la distance parcourue, ces mouvements sont relativement peu importants par rapport à d'autres du même genre qui ne concernent pas des lieux habités. Ils ne sont pas comparables aux anciens mouvements de grande ampleur répertoriés dans le secteur. Cette étude a donc permis d'analyser une situation qui existe depuis longtemps et qui semble à présent ne pas inquiéter les habitants du village. Ceux ci, conscients des possibles réactivations futures, mais considérant les ressources hydriques à proximité du village préfèrent s'accommoder des risques d'éboulements. La prévention pourrait être améliorée par une mise sous surveillance du site par GPS (Global Positioning System) pour quantifier les déplacements du pan rocheux et de l'escarpement sommital. De là, se définiraient les propositions susceptibles de réduire le risque au niveau du village.

\section{Bibliographie}

Durand-Delga M. - « La courbure de Gibraltar, extrémité occidentale des chaines alpines, unit l'Europe et I'Afrique 1. Ecologae. Geologica Helvetica, V. 65, n"2, 1972, p. 267-278

Durand-Delga M., Hottinger L., Marcais J., Milliard Y., Suter G. - " Données actuelles sur la structure du Rif $n$. Mémoire hors série. Société Géologique de France, t. I, 1962, p. 399-422.

El Mrihi A. - Structures alpines des zones externes et des nappes des flvschs à louest de la chaine du Haouz (Rif septentrional. Maroc). These de $3^{\circ}$ cycle, Université Mohamed V, Rabat, 1995, 155 p.
El Kharim Y, Hlila R., Darraz. C. El Haijaii K. - " Ecroulements et mouvements associés dans le col d'El Onsar (Rif, Marocl n. Proceeding of the II Workshop on Seismic Risk in North Africa, Tétouan, 2001, p, 43-46.

Fallot P. - " Essaî sur la géologie du Rif septentrional n. Notes et Mémoire Service Géologique du Maroc, 40, 1937, $553 \mathrm{p}$.

Hlila R. - Étude des déformations miocènes dans la chaine calcaire du Haouz lentre Tétouan et le col du Drauy). Thèse de $3^{\circ}$ cycle, Université Abdelmalek Essaadi, Tẻtouan, 1994, 159 p.
Maate A. - Estratigrafía y evolución paleoqeográfica alpina del dominio gomâride (Rif interno, Maruecos). Thèse de doctorat, Universidad Granada, 1996, 397 p.

Margaa K. - Essai de cartographie des risques naturels : application à l'aménagement de la région d'Al Hoceima (Rif. Nord-Maroc). Thèse de doctorat, Université de Franche-Comté, 1994, 196 p.

MOPT - Ortoimagen a 1/100 000 del Estrecho de Gibraltar. I.N.G. España, MOPT,

Raoult J.F - La chaîne du Haouz du col d'Azlus d'Arbia à Bab Aonsar. Notes et Mémoires. Service Géologique du Maroc, $n^{*} 184,1966$, p. $61-148$. 